\title{
Bidirectional 2.5-Gb/s WDM-PON Using FP-LDs Wavelength-Locked by a Multiple-Wavelength Seeding Source Based on a Mode-Locked Laser
}

\author{
Quoc Thai Nguyen, Student Member, IEEE, Pascal Besnard, Member, IEEE, Laurent Bramerie, Alexandre Shen, \\ Christophe Kazmierski, Philippe Chanlou, Guang-Hua Duan, Senior Member, IEEE, and Jean-Claude Simon
}

\begin{abstract}
We experimentally investigate the operation of a cost-effective wavelength-division-multiplexed passive optical network (WDM-PON) based on wavelength-locked Fabry-Pérot laser diodes (FP-LDs). A single quantum-dash passively mode-locked laser (QD-MLL) is combined with an arrayed waveguide grating in WDM-PON architecture to provide a low-noise, coherent multiwavelength seeding source to injection-lock the FP-LDs for both downstream and upstream. The results show that the QD-MLL-injected FP-LD has the same performance when compared to the case of injection-locking by a low-noise external cavity laser. Error-free bidirectional transmission over $25 \mathrm{~km}$ for 16 channels with 42.7-GHz channel spacing is demonstrated at $2.5 \mathrm{~Gb} / \mathrm{s}$ in the $C$-band and an optical budget higher than $30 \mathrm{~dB}$ is reached.
\end{abstract}

Index Terms-Colorless operation, injection-locked Fabry-Pérot laser (IL-FP), wavelength-division-multiplexed passive optical network (WDM-PON), wavelength-locking.

\section{INTRODUCTION}

$\mathbf{T}$ O MEET the ever-increasing demand of bandwidth, wavelength-division-multiplexed passive optical network (WDM-PON) has been considered for a long time as one of the most powerful solutions for the next-generation broadband access network. Recently, substantial research efforts have been investigated to provide low-cost and wavelength-independent (so-called colorless) transmitters for WDM-PON. Some well-known solutions are generally based on the use of a Fabry-Pérot laser diode (FP-LD) [1] or a reflective semiconductor optical amplifier (RSOA) [2] externally injected by a spectrum-sliced broadband light source (BLS). However, since spectral slicing of a BLS inherently suffers from strong intensity noise [1], [3] as well as incoherent characteristic, the transmission is difficult to be achieved at $2.5 \mathrm{~Gb} / \mathrm{s}$. There are

Manuscript received September 18, 2009; revised February 15, 2010; accepted February 22, 2010. Date of publication March 15, 2010; date of current version April 30, 2010. This work was supported by the French National Research Agency in the frame of the project ANTARES.

Q. T. Nguyen, P. Besnard, L. Bramerie, and J.-C. Simon are with the CNRS FOTON Laboratory (UMR 6082), ENSSAT, Université de Rennes 1, 22305 Lannion, France (e-mail: quocthai.nguyen@enssat.fr; pascal.besnard@enssat.fr; laurent.bramerie@enssat.fr; Jean-Claude.Simon@enssat.fr).

A. Shen, C. Kazmierski, and G.-H. Duan is with the Alcatel-Thales III-V Laboratory, a joint laboratory of Alcatel-Lucent Bell Laboratories and Thales Research and Technologies, 91461 Marcoussis, France (e-mail: alexandre.shen@3-5lab.fr; christophe.kazmierski@3-5lab.fr; guang-hua.duan@3-5lab.fr).

P. Chanclou is with Orange Laboratories, 22307 Lannion, France (e-mail: philippe.chanclou@orange-ftgroup.com).

Color versions of one or more of the figures in this letter are available online at http://ieeexplore.ieee.org.

Digital Object Identifier 10.1109/LPT.2010.2044569 several demonstrations at $2.5 \mathrm{~Gb} / \mathrm{s}$ based on spectral slicing of BLS [4] or a conventional Fabry-Pérot laser [5], but their reported performances are limited; a bidirectional transmission capability over a typical length of access network (20 km or more) has not been achieved.

Recently, quantum-dash mode-locked lasers have been the subject of many investigations owing to their remarkable properties [6]. Particularly, it can be used as a wavelength comb for WDM sources [7]. This application is more cost-effective when compared to the use of a set of single-mode lasers. We take advantage of such benefits to propose a WDM-PON architecture that is based upon a multiwavelength source, optically injected by a quantum-dash Fabry-Pérot passively mode-locked laser (QD-MLL) with 42.7-GHz mode spacing. The injection-locked Fabry-Pérot laser diode (IL-FP) directly modulated at $2.5 \mathrm{~Gb} / \mathrm{s}$ acts as a colorless transmitter. This proposal is extremely interesting in order to overcome the limitations of recently used spectral-slicing techniques. Previously, we preliminarily demonstrated this concept for WDM-PON [8]. However, the insufficient optical gain and the low locking-sensitivity of our IL-FP required a high required injected power. It implied that the single-fiber upstream (US) transmission was not achieved because of the limitation due to the Rayleigh backscattering. Consequently, the WDM-PON configuration and the system performances were limited to a dual-fiber architecture with a low available optical budget and a low number of channels. With the improvement of the optical gain and the locking-sensitivity of IL-FP, we demonstrated in this letter its feasibility for a single-fiber WDM-PON architecture over $25 \mathrm{~km}$ supporting 16 channels with $42.7 \mathrm{GHz}$ interspacing in the $C$-band.

\section{QD-MLL-INJECTED FP-LD COLORLESS TRANSMITTER}

The InAs-InP-based QD-MLL under study is mode-locking at a frequency of $42.7 \mathrm{GHz}$. As shown in Fig. 1, its optical spectrum is very wide and almost flat over a spectral range of $14 \mathrm{~nm}$ covering 40 modes (spectral power flatness of $4 \mathrm{~dB}$ ). Sixteen modes ranging from 1548.70 to $1553.84 \mathrm{~nm}$ have been chosen as seeding wavelengths for downstream (DS) and 16 modes ranging from 1556.96 to $1562.15 \mathrm{~nm}$ for US. The DS and US bands are separated by a guard band of $3 \mathrm{~nm}$ covering eight modes. Since the mode spacing is not compatible with the standardized WDM channel spacing, a tunable arrayed waveguide grating (AWG) is used. It is adjusted in frequency and in channel spacing so that its channel grid coincides with that of QD-MLL modes. One individual mode is then selected by a specified channel of the AWG. For practical implementation, a 


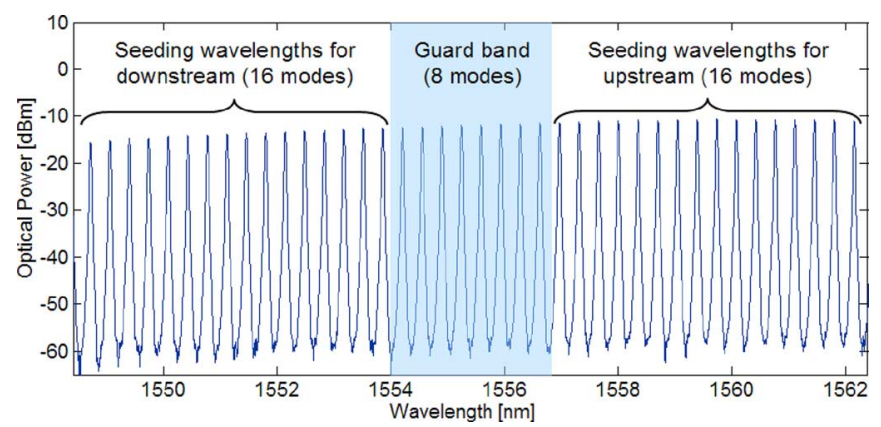

Fig. 1. Optical spectrum of QD-MLL at 300-mA bias.
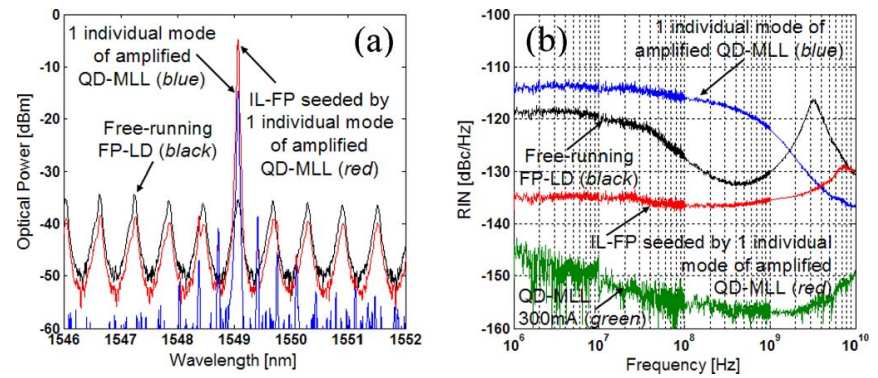

Fig. 2. (a) Injection-locking spectra and (b) RIN.

mode spacing compatible with the standardized WDM channel spacing could be achieved by optimizing the cavity length of our QD-MLL [7].

The injection-locking spectra are given in Fig. 2(a). One individual mode of QD-MLL amplified with an erbium-doped fiber amplifier (EDFA) is selected by the AWG and used for optical injection. The power of this individual mode is fixed at $-5 \mathrm{dBm}$. Under injection, the FP-LD is locked to the wavelength of the injected signal and is running in a single-mode operation with a sidemode suppression ratio (SMSR) higher than $30 \mathrm{~dB}$.

The FP-LD was designed to be polarization-insensitive under injection-locking thanks to the superimposition of TE and TM modes. The operation principle of polarization-insensitive IL-FP was reported in [9]. However, since the gains of TE and TM modes are slightly different, the polarization-insensitivity is not completely reached. Consequently, it gives rise to small variations on the level " 1 " of modulated laser when the polarization state of the injected signal is changed. These variations become stronger when the injected power is low $(<-10 \mathrm{dBm})$, which is usually the case of IL-FP at the optical network unit (ONU). The second feature of two sections FP-LD is that their wavelength is tunable, by varying the biased current of the second section, in order to have an optimal detuning for which a maximum injection-locking efficiency is obtained.

A major inconvenience when using a wavelength comb of QD-MLL as a WDM source is, in comparison to the single-mode laser, the increase of relative intensity noise (RIN) of the selected mode due to mode partition noise [8], particularly in low frequency. Fig. 2(b) shows RIN. The RIN rises up to $-115 \mathrm{dBc} / \mathrm{Hz}$ at low frequency. However, thanks to the injection-locking, the RIN is strongly reduced to less than $-130 \mathrm{dBc} / \mathrm{Hz}$ because the IL-FP is forced to new lasing regime with a higher resonance frequency, as observed in Fig. 2(b).

Fig. 3 gives a comparison between two cases of injectionlocking at the same injection conditions $(-5 \mathrm{dBm}$ of injected power at $1556.96 \mathrm{~nm}$ ): one using a selected mode of QD-MLL
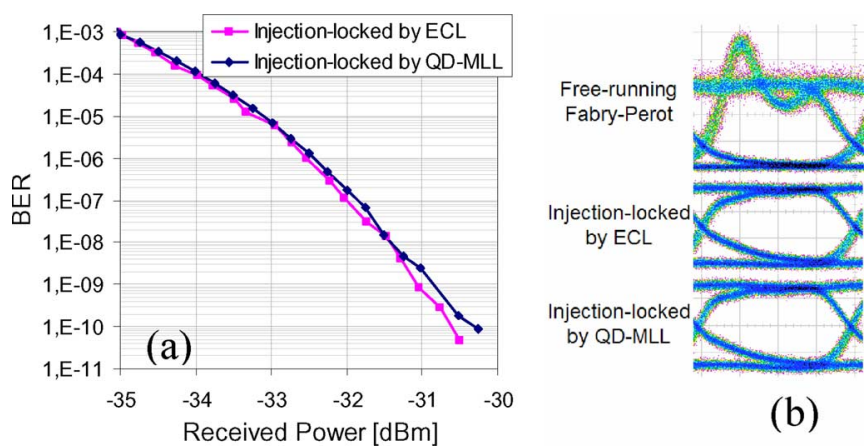

Fig. 3. Comparison of BER measurement for two seeding sources (a) and corresponding measured eye diagrams at $2.5 \mathrm{~Gb} / \mathrm{s}$ modulation (b).
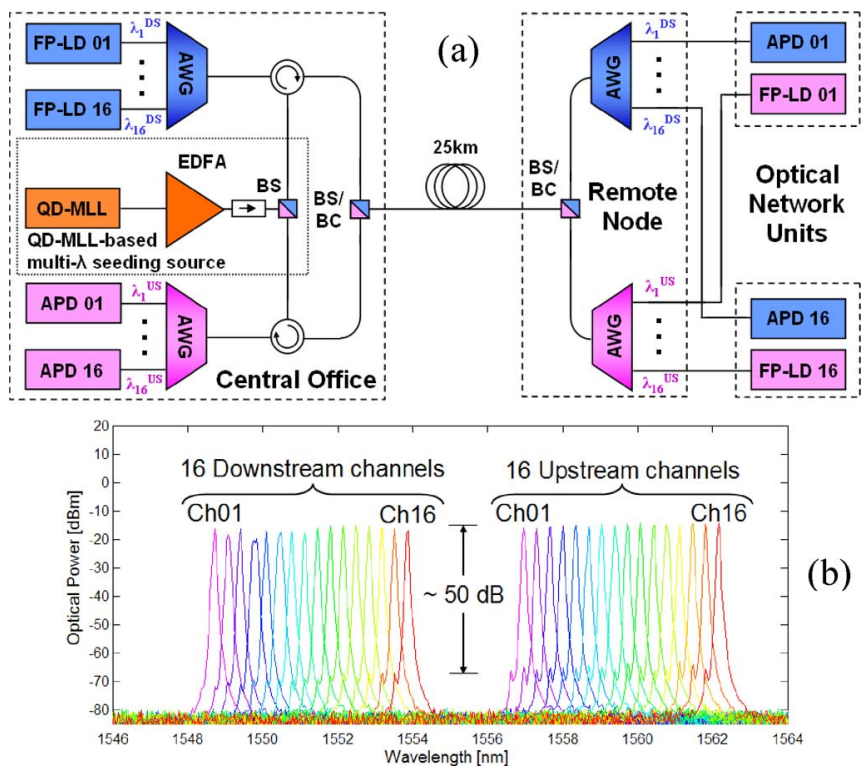

Fig. 4. Schematic of proposed bidirectional WDM-PON architecture (a) and wavelength allocation of 16 WDM channels for DS and US transmissions (b).

and another using a low-noise external cavity laser (ECL). It is found that the performances are quite comparable in terms of bit-error rate (BER) as shown in Fig. 3(a). It is confirmed in Fig. 3(b) by eye diagrams. The difference between free-running FP-LD and IL-FP is linked to relaxation frequency enhancement and to intensity-noise reduction [Fig. 2(b)].

\section{BIDIRECTIONAL WDM-PON ARCHITECTURE AND EXPERIMENTAL PERFORMANCES}

The bidirectional single-fiber architecture is schematized in Fig. 4(a). The amplified QD-MLL is used as a multiwavelength seeding source for both DS and US. The separation/combination of DS and US bands are performed by the band separator/combiner (BS/BC). The transmission medium consists of $25 \mathrm{~km}$ of single-mode fiber. Fig. 4(b) shows the wavelength allocation of 16 WDM channels for DS and US transmission. Since each channel is filtered two times by AWG, the SMSR of the received signal is about $50 \mathrm{~dB}$.

Since the DS and US signals operate on the two separated wavelength bands, there is no significant impact between DS and US transmissions. Consequently, the experimental performances of bidirectional WDM-PON could be reasonably evaluated for separate DS and US transmissions. The QD-MLL is biased at high current $(300 \mathrm{~mA})$ in order to have a lower 

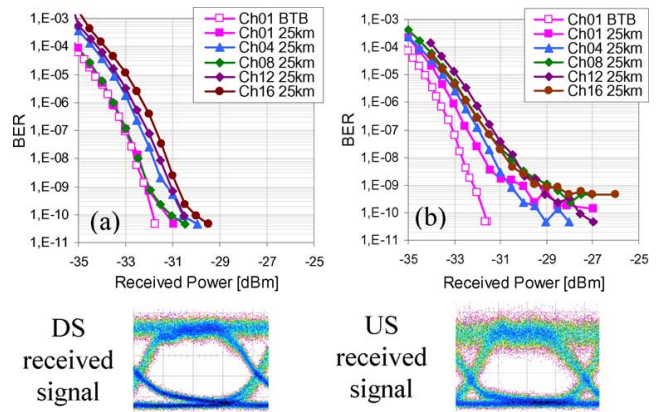

Fig. 5. BER measurement results for DS (a) and US (b) transmissions and the corresponding eye diagrams.

intensity noise. It is then amplified by an EDFA with an output power of $20 \mathrm{dBm}$. Two commercial tunable AWGs are adjusted so that their channels match the selected modes of QD-MLL. The FP-LD is biased at $65 \mathrm{~mA}$ for the gain section and has an output power of $+2 \mathrm{dBm}$. The second section is biased above a threshold current of $18 \mathrm{~mA}$, in order to achieve the superposition of TE and TM modes over the operating wavelength range, and is chosen up to $100 \mathrm{~mA}$ so that an optimal wavelength-detuning is obtained for a good injection-locking efficiency. The gain section is then directly modulated with a 2 -Vpp amplitude and $2^{31}-1$ PRBS at $2.5 \mathrm{~Gb} / \mathrm{s}$. At the reception, the signal is directly detected by an avalanche photodiode (APD) having $2.5-\mathrm{GHz}$ bandwidth. With this configuration, the injected optical power in the FP-LD is $-2 \mathrm{dBm}$ in the case of DS transmission and $-10 \mathrm{dBm}$ for US transmission. For these injected powers, the polarization-insensitivity of injection-locking is completely achieved for DS transmission while there are still several instabilities in the case of US transmission due to the polarization variation.

The measurements of BER versus received power for DS and US transmission are shown in Fig. 5(a) and (b), respectively. For clarity, only five WDM channels (Ch01, Ch04, Ch08, Ch12, Ch16) out of 16 are shown. These results show a good homogeneity in performances of the different channels. Error-free transmission is achieved for all of these channels. For DS transmission, a negligible power penalty is observed when compared to the performance of back-to-back (BTB) configuration with one after $25-\mathrm{km}$ transmission for the Ch01. A sensitivity around $-31 \mathrm{dBm}$ is obtained at $10^{-9} \mathrm{BER}$ for the DS transmission. For US, since the single-fiber architecture is used, the performance of bidirectional transmission (of DS CW seeding signal and US data on the same wavelength) is impaired by interferometric noise caused by the Rayleigh backscattering and the back-reflection [10]. This interferometric noise is illustrated in the eye diagram of US signal in Fig. 5. Consequently, a power penalty of $2 \mathrm{~dB}$ is obtained and an error floor between $10^{-9}$ and $10^{-10}$ is observed, as shown in Fig. 5(b).

A lower sensitivity of $-28 \mathrm{dBm}$ at $10^{-9}$ BER is found for the 16 US channels. As the FP-LD has an output power of $+2 \mathrm{dBm}$, this proposed system can support an optical budget of at least $30 \mathrm{~dB}$. A power budget analysis for the proposed WDM-PON system is shown in Table I. We can see that power margins of at least $10 \mathrm{~dB}$ are available for both DS and US.
TABLE I

OPTICAL BUDGET ANALYSIS FOR PROPOSED WDM-PON SYSTEM

\begin{tabular}{lcc}
\hline \hline \multicolumn{1}{c}{ Parameters } & Downstream & Upstream \\
\hline IL-FP output power [dBm] & +2 & +2 \\
AWG loss (2 stages) [dB] & 10 & 10 \\
Fiber attenuation [dB] & 5 & 5 \\
Circulator and connectors loss [dB] & 3 & 3 \\
Band separator / combiner (2 stages) [dB] & 2 & 2 \\
Received power [dBm] & -18 & -18 \\
$10^{-9}$ Receiv. sensitivity (worst case) $[\mathrm{dBm}]$ & -31 & -28 \\
Power margin [dB] & 13 & 10 \\
\hline \hline
\end{tabular}

\section{CONCLUSION}

The proposed concept and the demonstrated results in this letter show that QD-MLL could be a promising solution as low-noise, low-cost seeding source for IL-FP-based WDM-PON system. This solution can be also applied for colorless WDM-PON based on other reflective component technologies such as RSOA or REAM. Error-free bidirectional transmission of a total capacity of $40 \mathrm{~Gb} / \mathrm{s}$ is demonstrated for a simple and cost-effective WDM-PON architecture. An optical budget higher than $30 \mathrm{~dB}$ and an available power margin at least $10 \mathrm{~dB}$ are obtained. The use of a polarization-insensitive IL-FP is also reported. Further work will be investigated to increase the injection-locking sensitivity of FP-LD, to achieve a better polarization-insensitivity, and to increase the modulation bandwidth of IL-FP in order to match 10-Gb/s WDM access.

\section{REFERENCES}

[1] J. H. Lee, C. H. Kim, Y. G. Han, and S. B. Lee, "WDM-based passive optical network upstream transmission at $1.25 \mathrm{~Gb} / \mathrm{s}$ using Fabry-Perot laser diodes injected with spectrum-sliced, depolarized, continuouswave supercontinuum source," IEEE Photon. Technol. Lett., vol. 18, no. 20, pp. 2108-2110, Oct. 15, 2006.

[2] F. Payoux, P. Chanclou, M. Moignard, and R. Brenot, "Gigabit optical access using WDM PON based on spectrum slicing and reflective SOA," in Proc. ECOC '05, 2005, vol. 3, pp. 455-456.

[3] K.-Y. Park and C.-H. Lee, "Intensity noise in a wavelength-locked Fabry-Perot laser diode to a spectrum sliced ASE," IEEE J. Quantum Electron., vol. 44, no. 3, pp. 209-215, Mar. 2008.

[4] A. Borghesani, I. F. Lealman, A. Poustie, D. W. Smith, and R. Wyatt, "High temperature, colourless operation of a reflective semiconductor optical amplifier for $2.5 \mathrm{Gbit} / \mathrm{s}$ upstream transmission in a WDM-PON," in Proc. ECOC '07, 2007, pp. 641-642.

[5] Y.-S. Liao and G.-R. Lin, "22-channel detuning capacity of a side-mode injection locked FPLD for directly modulated $2.5 \mathrm{Gbit} / \mathrm{s}$ DWDM-PON," in Proc. OFC/NFOEC '07, 2007, Paper OMS8.

[6] F. Lelarge et al., "Recent advances on InAs/InP quantum dash based semiconductor lasers and optical amplifiers operating at $1.55 \mu \mathrm{m}$," IEEE J. Sel. Topics Quantum Electron., vol. 13, no. 1, pp. 111-124, Jan./Feb. 2007.

[7] A. Akrout et al., "Error-free transmission of 8 WDM channels at 10 $\mathrm{Gbit} / \mathrm{s}$ using comb generation in a quantum dash based mode-locked laser," in Proc. ECOC '08, Brussels, Belgium, Sep. 21-25, 2008, Paper Th3.D.2.

[8] Q. T. Nguyen et al., "Bidirectional transmission in colourless WDM-PON based on injection-locked Fabry-Perot laser at $2.5 \mathrm{Gbit} / \mathrm{s}$ using low-cost seeding source," in Proc. ECOC '09, Vienna, Austria, Sep. 20-24, 2009, Paper 6.5.1.

[9] A. Shen et al., "Polarization insensitive injection locked Fabry-Perot laser diodes for $2.5 \mathrm{~Gb} / \mathrm{s}$ WDM access applications," in Proc. ECOC '08, Brussels, Belgium, Sep. 21-25, 2008, Paper Th.3.D.1.

[10] M. Fujiwara, J. I. Kani, H. Suzuki, and K. Iwatsuki, "Impact of backreflection on upstream transmission in WDM single-fiber loopback access networks," J. Lightw. Technol., vol. 24, no. 2, pp. 740-746, Feb. 2006. 